\title{
Staatliche Risikosteuerung und Verfassung
}

Werner Heun"

A. Einleitung: Sicherheit - Gefahr -

Risiko ............................ 376

I. Sicherheit ....................... 376

II. Gefahr........................... 377

III. Juristischer Risikobegriff .............. 378

IV. Weiter Risikobegriff ............... 380

B. Methoden der Risikosteuerung ........ 382

I. Risikoreduzierung ................. 382

II. Risikoverlagerung ................ 382

III. Risikoverteilung ................... 383

C. Probleme der Risikosteuerung durch

den privaten Sektor .................. 384

I. Asymmetrische Information........ 384
II. Risikowahrnehmung.............. 386

III. Verpflichtungsprobleme ............ 389

IV. Externalitäten .................... 391

D. Die Rolle des Staates als ultimativer

Risikomanager ..................... 392

E. Verfassungsrechtliche Direktiven für die staatliche Risikosteuerung ........ 395

I. Risikosteuerung als Eingriffsrechtfertigung......................... 395

II. Verfassungsrechtliche Pflicht zur Risikosteuerung? ................... 395

III. Geringe Determinationskraft der Verfassung ........................ 397

\section{A. Einleitung: Sicherheit - Gefahr - Risiko}

Auf den ersten Blick ist staatliche Risikosteuerung im deutschen Verfassungsrecht ein wenig vertrautes Konzept. Demgegenüber sind die Konzepte der Sicherheit und ihrem Gegenteil, der Gefahr, wesentlich bekannter und verbreiteter. Die drei Begriffe der Sicherheit, der Gefahr und des Risikos sind jedoch eng miteinander verbunden und die Risikosteuerung ist dementsprechend schon seit langem eine Staatsfunktion, obwohl sie noch nicht hinreichend als solche erfasst und begriffen worden ist. Bevor man sich dem Thema der Risikosteuerung selbst zuwendet, erscheint es sinnvoll, die drei Begriffe und ihr Verhältnis zueinander in einem kurzen historischen Überblick zu klären.

\section{Sicherheit}

Das älteste Konzept in der politischen Philosophie wie auch in der Verfassungstheorie ist die Sicherheit. ${ }^{1}$ Der römische Begriff der securitas, von dem alle modernen europäischen Begriffsbildungen abgeleitet sind, bedeutet ursprünglich schlicht die Abwesenheit von Schmerz und Unwohlsein. Er gewann politische Bedeutung als ein Ausdruck für die Pax Romana, bedeutete aber lediglich ein subjektives Gefühl, keinen objektiven Zustand. Während des Mittelalters erhielt der Begriff der securitas allerdings immer mehr einen objektiven Gehalt und wurde in ein positives politisches

* Prof. Dr. Dr. h.c. Werner Heun ist Leiter des Instituts für Allgemeine Staatslehre und Politische Wissenschaften der Georg-August-Universität Göttingen. Der Beitrag ist die wesentlich erweiterte und ergänzte deutsche Fassung eines englischen Vortrags: Risk Management by the Government and the Constitution, in: Gunnar Duttge/San Won-Lee (Hrsg.), The Law in the Information and Risk Society, 2011, S. 15.

1 Historischer Überblick bei W. Conze, Sicherheit, Schutz, in: O. Brunner/W. Conze/R. Koselleck (Hrsg)., Geschichtliche Grundbegriffe, Bd. 5, Stuttgart 1984, S. 831-862; A. Schrimm-Heins, Gewissheit und Sicherheit. Geschichte und Bedeutungswandel der Begriffe certitudo und securitas, Archiv für Begriffsgeschichte 34 (1991), S. 123-213; 35 (1992), S. 115-213; M. Makropoulos, in: J. Ritter/K. Gründer/G. Gabriel (Hrsg.), Historisches Wörterbuch der Philosophie, Bd. 9, Basel 1995, Sp. 745-750. 
Konzept verwandelt. Erst die Entstehung des modernen Staates führte allerdings dazu, dass securitas publica zum vorrangigen Ziel und Zweck des Staates avancierte. ${ }^{2}$

Dieser allgemeine Staatszweck wird in zwei verschiedene Aspekte aufgeteilt: Die innere und die äußere Sicherheit, die von verschiedenen Regeln und Maximen geleitet und beherrscht werden. ${ }^{3}$ Thomas Hobbes integriert Sicherheit in eine kohärente politische Theorie, indem er sie definiert als „not mere survival in any condition but a happy life so far as that is possible". ${ }^{4}$ John Locke qualifiziert Sicherheit schließlich als zentrales Element eines einheitlichen Staatsziels des „peace, safety and publick good of the people", 5 die ihrerseits eine Konkretisierung von life, liberty and property als Staatszweck darstellen. ${ }^{6}$ Im 18. Jahrhundert wurde die öffentliche Sicherheit zu einem umfassenden Konzept ausgeweitet, das die Wohlfahrt - in altertümlicher Wendung die Glückseligkeit - des Volkes einschloss. ${ }^{7}$ Im Gegenzug wurde die Sicherheit vom Liberalismus auf ihren Kern als öffentliche Sicherheit in einem engen Sinn sowie auf den Gedanken der Rechtsstaatlichkeit reduziert. ${ }^{8}$ Am Ende des 19. Jahrhunderts setzte schließlich ein erneuter Prozess der Ausweitung ein. Die Idee der sozialen Sicherheit wurde nunmehr zum vorherrschenden und dominierenden Ziel des Staates, ${ }^{9}$ schon bevor der Ausdruck von F.D. Roosevelt in den 1930er Jahren verwendet wurde ${ }^{10}$ und seitdem allgemein gebräuchlich wurde.

\section{Gefahr}

Sicherheit ist immer von Gefahren bedroht. Im Allgemeinen bedeutet Gefahr, dass ein Schaden droht. Als juristisches Konzept ist der Begriff der Gefahr ein Produkt des deutschen Polizeirechts, der bereits im 19. Jahrhundert entwickelt worden ist ${ }^{11}$

2 Vgl. a. J. Isensee, Das Grundrecht auf Sicherheit, Berlin et al. 1983, S. $3 \mathrm{ff}$.

3 Conze, Sicherheit (Fn. 1), S. 842 f.

4 T. Hobbes, De cive (1647), ch. 13, 4. (englische Ausgabe hrsg. von R. Tuck/M. Silverthorne, Cambridge, UK: Cambridge University Press 1998) S. 143; idem, Leviathan (1651), II, 30.

5 John Locke, Two Treatises of Government (1690), II, ch. 9, \$123 ff., 131.

6 P. Graf Kielmannsegg, Volkssouveränität, Stuttgart 1977, S. 143; W. Euchner, Naturrecht und Politik bei John Locke, Frankfurt am Main 1969 (Nachdr. 1979), S. 198 ff.

7 So z.B. C. Wolff, Vernünftige Gedancken von dem Gesellschaftlichen Leben der Menschen und insonderheit dem gemeinen Wesen (4. Aufl. 1736), II, ch. 1, $\$ 222$ f., in: C. Wolff, Gesammelte Werke 1. Abt. Bd. 5, Hildesheim/New York 1975, S. 165 f.

8 I. Kant, Über den Gemeinspruch: Das mag in der Theorie richtig sein, taugt aber nicht für die Praxis (1793), A 232-270, in: Werke (Hrsg. W. Weischedel), Bd. 6, S. 127-172 (143 ff.); W. von Humboldt, Ideen zu einem Versuch, die Grenzen der Wirksamkeit des Staates zu bestimmen (1792), in: Werke (Hrsg. von A. Flitner/K. Giel), Bd. 1, Darmstadt 1960, S. 56-233.

9 Vgl. F.-X. Kaufmann, Sicherheit als soziologisches und sozialpolitisches Problem, 2. Aufl., Stuttgart 1973, S. 91 ff.; G. A. Ritter, Der Sozialstaat, 2. Aufl., München 1991; zum deutschen Konzept des Sozialstaats vgl. H. Zacher, Das soziale Staatsziel, in: Handbuch des Staatsrechts, Bd. II, 3. Aufl., Heidelberg 2004, $\mathbb{2} 28$, S. 659-784.

10 F. D. Roosevelt, Speech 30. Sept. 1934, The Public Papers and Addresses of Franklin D. Roosevelt (Hrsg. S.I. Rosenman), Vol. 3, New York 1938, S. 413-425 (421).

11 Grundsatzurteil: Preußisches Oberverwaltungsgericht vom 10. Juni 1880, in: Preuß. Verwaltungsblatt $1879 / 80$, S. $401 \mathrm{ff}$. 
und nach einer gebräuchlichen Definition eine Situation oder Bedingung bedeutet, bei der im konkreten Fall die hinreichende Wahrscheinlichkeit besteht, dass in absehbarer Zeit ein Schaden für die öffentliche Sicherheit oder Ordnung eintreten wird. ${ }^{12}$ Diese von preußischen Verwaltungsgerichten entwickelte Formel findet auch heute noch immer Anwendung. Da die öffentliche Sicherheit in diesem Kontext die gesamte Rechtsordnung umfasst, nach einer gängigen Definition nämlich die objektive Rechtsordnung, die Rechte des Einzelnen sowie Einrichtungen des Staates, bedeutet jede Verletzung eines öffentliche Interessen schützenden Rechts zugleich eine Verletzung der öffentlichen Sicherheit. ${ }^{13}$ Sofern eine konkrete Gefahr droht, die durch die hinreichende Wahrscheinlichkeit des Schadenseintritts aus Sicht der handelnden Behörde definiert ist, ${ }^{14}$ sind die Behörden einschließlich der Polizei zur Ergreifung der notwendigen Maßnahmen ermächtigt und gegebenenfalls auch verpflichtet. Die hohe Wahrscheinlichkeit der Gefahrverwirklichung ist in diesem Kontext die entscheidende Differenz zum (bloßen) Risiko. In diesem Sinn ist auch die Gefahrenabwehr durch den Staat von staatlicher Risikosteuerung zu unterscheiden.

\section{Juristischer Risikobegriff}

Konzept und Begriff des Risikos sind dagegen erst eine neuere Entwicklung im deutschen Recht. Das traditionelle Konzept der Gefahrenabwehr nach polizeirechtlichen Grundsätzen wurde zunehmend als unzureichend angesehen, da die moderne Technik, insbesondere der Betrieb der Atomkraftwerke, zu katastrophalen Schäden führen kann, obwohl der Schadenseintritt äußerst unwahrscheinlich ist. Diese Risikokategorie wird daher im Englischen prägnant als low probability, high consequence risk bezeichnet. Insofern wurde die polizeirechtliche Gefahrenabwehr durch das neue Konzept der Risikovorsorge ergänzt. In diesem Zusammenhang wird Risiko rechtlich definiert als ein Produkt des (hohen) Ausmaßes des erwarteten Schadens und der (geringen) Wahrscheinlichkeit des Schadenseintritts. Die maßgebende Differenz zur konkreten Gefahr ist - allein und ausschließlich - die wesentlich geringere Eintrittswahrscheinlichkeit. ${ }^{15}$ Insofern gewichtet der Risikobegriff Schaden und Wahrscheinlichkeit anders als der Gefahrenbegriff. Eingebettet ist dieser Risikobegriff in ein Dreistufenmodell, das zwischen Gefahr, Risiko und Restrisiko differenziert, die nach Wahrscheinlichkeitsgrad voneinander abgegrenzt werden und verschiedene Rechts-

12 So z.B. $\$ 2$ Nr. 1 a Nds. SOG; F.-L. Knemeyer, Polizei- und Ordnungsrecht, 11. Aufl., München 2007, S. 62 ff.; V. Götz, Allgemeines Polizei- und Ordnungsrecht, 14. Aufl., München 2008, $\mathbb{} 4$ Rn. 1 ff.

13 Knemeyer, Ordnungsrecht (Fn. 12), S. $72 \mathrm{f}$.

14 B. Drews/G. Wacke/K.Vogel/W. Martens, Gefahrenabwehr, 9. Aufl., München 1986, S. 224; R. Poscher, Gefahrenabwehr, Berlin 1999, S. $114 \mathrm{ff}$.

15 Ursprüngliche Definition: BMFT (Hrsg.), Deutsche Risikostudie Kernkraftwerke 1979, Hauptband, S. $10 \mathrm{ff}$.; vgl. ferner J. Ipsen, Die Bewältigung der wissenschaftlichen und technischen Entwicklungen durch das Verwaltungsrecht, VVDStRL 48 (1990) S. 177-206 (186f.); A. Reich, Gefahr - Risiko Restrisiko, Düsseldorf 1989, S. 85 ff.; U. di Fabio, Risikoentscheidungen im Rechtsstaat, Tübingen 1994, S. 73 f.; L. Jaeckel, Gefahrenabwehrrecht und Risikodogmatik, Tübingen 2010, S. 49 ff.; kritisch A. Scherzberg, Risiko als Rechtsproblem, Verwaltungsarchiv 84 (1993), S. 484-513 (497 ff.). 
folgen auslösen. Eine (konkrete) Gefahr ist prinzipiell abzuwehren, Risiken sind im Sinne der Risikovorsorge zu minimieren, während das Restrisiko hinzunehmen ist. ${ }^{16}$ Diesem Dreistufenmodell wird seit längerem ein Zweistufenmodell gegenübergestellt, das Risiko als Oberbegriff und die Möglichkeit des Schadenseintritts als Definitionsmerkmal des Risikos ansieht. ${ }^{17}$ Dabei wird angenommen, dass der Risikobegriff erst durch das Möglichkeitskriterium sich vom Gefahrenbegriff, der weiterhin durch die Wahrscheinlichkeit definiert sein soll, emanzipiert und eigenständige Qualität gewonnen habe. ${ }^{18}$ Das ist freilich ein kategoriales Missverständnis, da Wahrscheinlichkeit und Möglichkeit prinzipiell dasselbe bezeichnen. Wahrscheinlichkeit ist nämlich lediglich die Größe bzw. das Maß der Möglichkeit. ${ }^{19}$

Diese Risikodiskussion ist allerdings im Wesentlichen auf das Recht der technischen Sicherheit beschränkt. ${ }^{20}$ Sogar der weit gefasste Begriff der Risikogesellschaft bezieht sich in erster Linie auf diese technischen Risiken. ${ }^{21}$ Die hauptsächlichen Anwendungsfelder sind das Atomrecht, die Bereiche der Gentechnologie und in jüngster Zeit der Nanotechnologie. ${ }^{22}$ In diesen Bereichen dient Risikovorsorge als Grundlage rechtlicher Abwehrmaßnahmen, die den Eintritt unmittelbarer und konkreter Gefahren im Voraus abwenden sollen. Das Prinzip der Risikovorsorge ermächtigt den

16 Vgl. hier nur BVerfGE 49, 89 (137-143); 53, 30 (59); R. Breuer, Gefahrenabwehr und Risikovorsorge im Atomrecht, DVBl. 1978, S. 829 ff. (835 f.); ders., Anlagensicherheit und Störfälle, NVwZ 1990, S. $211 \mathrm{ff}$. (213f.); H.-H. Trute, Staatliches Risikomanagement im Anlagenrecht. Länderbericht Deutschland, in: E. Riedel (Hrsg.), Risikomanagement im öffentlichen Recht, Baden-Baden 1997, S. 55-109 (93 ff.); jüngst A. G. Debus, Strategien im Umgang mit sagenhaften Risikotypen, insbesondere am Beispiel der Kernenergie, in: J.Scharrer/M. Dalibor/K. Rodi/K. Fröhlich/P. Schächterle (Hrsg.), Risiko im Recht - Recht im Risiko, Baden-Baden 2011, S. 11-36 (15 ff.).

17 M. Kloepfer/E. Rehbinder/E. Schmidt-Aßmann/P. König, Umweltgesetzbuch - Allgemeiner Teil, Berlin 1990, $\mathbb{2}$ Abs. 6; sowie dazu ebd. S.119f. in Anknüpfung an D. Murswiek, Die staatliche Verantwortung für die Risiken der Technik, Berlin 1985, S. 85; s. ferner z.B. C. Calliess, Rechtsstaat und Umweltstaat, Tübingen 2001, S. 162 ff.; Trute, Risikomanagement (Fn. 16), S. 98.

18 O. Lepsius, Risikosteuerung durch Verwaltungsrecht, VVDStRL 63 (2004), S. 264 ff. (268ff.); s.a. Calliess, Rechtsstaat (Fn. 17), S. 163; C. Transfeld, Das Vorsorgeprinzip im Lichte der ökonomischen Analyse des Rechts, Bayreuth 2006, S. 48 ff.

19 Vgl. Sondergutachten des Rates von Sachverständigen für Umweltfragen, Umwelt und Gesundheit Risiken richtig einschätzen, BT-Drs. 14/2300, S. 49. M.a.W.: Wenn die Möglichkeit eines Schadenseintritts besteht, besteht auch eine - wenigstens minimale - Wahrscheinlichkeit. Bei der Wahrscheinlichkeit $=0$ ist der Schadenseintritt auch nicht möglich. Risiko und Gefahr lassen sich daher in diesem Modell überhaupt nicht voneinander abgrenzen, sondern sind identisch. Zwar läßt sich das Risiko als Oberbegriff qualifizieren, die Gefahr kann aber weiterhin nur durch den erhöhten Grad der Wahrscheinlichkeit bestimmt werden. Im übrigen folgt aus der Umstellung auf die Möglichkeit nichts für die Drei- oder Zweistufigkeit der Rechtsfolgen, vgl. auch R. Wahl/I. Appel, Prävention und Vorsorge: Von der Staatsaufgabe zur rechtlichen Ausgestaltung, in: R. Wahl (Hrsg.), Prävention und Vorsorge, Berlin 1995, S. 1-216 (84 ff.).

20 Daneben aber etwa auch im Arzneimittelrecht, dazu di Fabio, Risikoentscheidungen (Fn. 15), S. 166 ff.; und im Lebensmittelrecht, dazu Transfeld, Vorsorgeprinzip (Fn. 18), S. 95 ff.

21 U. Beck, Risikogesellschaft, Frankfurt am Main 1986, S. 25-112; P. Lagadec, Das Grosse Risiko. Technische Katastrophen und gesellschaftliche Verantwortung, Nördlingen 1987 (frz. 1981/86), S. 22, 71 ff.; s.a. G. Bechmann, Risiko als Schlüsselkategorie der Gesellschaftstheorie, KritV 1991, S. 212-240, auch in: ders. (Hrsg.), Risiko und Gesellschaft, Opladen 1993, S. 237-276.

22 Vgl. Jaeckel, Gefahrenabwehrrecht (Fn. 15), S. $16 \mathrm{ff}$. 
Staat, entsprechende Präventionsmaßnahmen zu ergreifen. ${ }^{23}$ Mit dieser Darstellung der drei miteinander verbundenen Konzepte von Sicherheit, Gefahr und Risiko ist der gegenwärtige Stand der juristischen Diskussion zunächst einmal umschrieben.

\section{Weiter Risikobegriff}

Risiko und seine Steuerung ist jedoch ein Problem von sehr viel größerer Reichweite, das die engen Grenzen des technischen Risikokonzepts deutlich überschreitet, ${ }^{24} \mathrm{da}$ technische Risiken nur ein enger Teilbereich von Risiko überhaupt sind. Risiko in dem hier verstandenen, weiten Sinne weist zwei Elemente auf. ${ }^{25}$ Risiko setzt erstens eine Ungewissheit über die Zukunft voraus und zweitens die Möglichkeit eines irgendwie gearteten Schadenseintritts auf der einen Seite sowie auch meistens - wenn nicht immer - die Möglichkeit eines Gewinns oder anderer positiver Entwicklungen auf der anderen Seite. Als eine allgemeine Beobachtung kann man festhalten, dass nachteilige Eventualitäten nicht existieren können, ohne dass sie von vorteilhaften Eventualitäten begleitet werden. ${ }^{26}$ Naturkatastrophen scheinen auf den ersten Blick ein Gegenbeispiel darzustellen. Das ist aber nur der Fall, wenn man die Tatsache vernachlässigt, dass der Mensch das Risiko, von einer solchen Katastrophe getroffen zu werden, dadurch (bewusst) eingeht, indem er sich in Erwartung bestimmter Vorteile etwa an der Küste (im Fall von Überflutungen und Tsunamis) oder in einem Gebiet, das von Erdbeben oder einem Vulkanausbruch bedroht ist, niederlässt.

Die Entwicklung eines solchen breiten und umfassenden Konzepts des Risikos kann bis in die italienische Renaissance zurückverfolgt werden, als im 15. Jahrhundert Seeversicherungen eingerichtet wurden. ${ }^{27}$ Frühe Vorläufer solcher Versicherungen

23 Für Deutschland s. U. K. Preuß, Risikovorsorge als Staatsaufgabe, in: D. Grimm (Hrsg.), Staatsaufgaben, Baden-Baden 1993, S. 523-551; W. Köck, Risikovorsorge als Staatsaufgabe, AöR 121 (1996), S.1-23; allgemein vergleichend C. R. Sunstein, Laws of Fear. Beyond the Precautionary Principle, Cambridge, UK: Cambridge University Press 2005, S. 15 ff.

24 Risikosteuerung wird in Deutschland nur in diesem engen Sinn verstanden, vgl. z.B. den Sammelband E. Riedel (Hrsg.), Risikomanagement (Fn. 16).

25 Vgl. allgemein N. Rescher, Risk. A Philosophical Introduction to the Theory of Risk Evaluation and Management, Lanham, Maryland: University Press of America 1983, S. 5 ff.; J. F. Yates/E. R. Stone, The risk construct, in: J. Frank Yates (Hrsg.), Risk-Taking Behavior, Chichester: John Wiley and Sons 1992, S. 1-25; J. Adams, Risk, LOndon: UCL Press 1995; O. Rammstedt, Risiko, Historisches Wörterbuch der Philosophie (Fn. 1) Bd. 8, 1992, Sp. 1045-1050; anderer Ansatz bei H. Münkler, Strategien der Sicherung: Welten der Sicherheit und Kulturen des Risikos. Theoretische Perspektiven, in: ders./ M. Bohlender/S. Meurer (Hrsg.), Sicherheit und Risiko, Bielefeld 2010, S. 11-33.

26 D. A. Moss, When All Else Fails. Government as the Ultimate Risk Manager, Cambridge, Mass.: Harvard University Press 2002, S. 22.

27 Vgl. P. Perdikas, Die Entstehung der Versicherung im Mittelalter, Zeitschrift für die gesamte Versicherungswissenschaft 55 (1966), S. 425-509; K. Nehlsen-von Stryk, Die venezianische Seeversicherung im 15. Jahrhundert, Ebelsbach 1986; K. H. Van D’Elden, The Development of the Insurance Concept and Insurance Law in the Middle Ages, in: H. J. Johnson (Hrsg.), The Medieval Tradition of Natural Law, Kalamazoo: Western Michigan University 1987, S. 191-199 (196f.); vgl. auch D. C. North, Institutions, Journal of Economic Perspectives 5 (1991), S. 97-112 (106 f.); J. Franklin, The Science of Conjecture. Evidence and Probability before Pascal, Baltimore: The Johns Hopkins University Press 2001, S. $273 \mathrm{ff}$. 
kann man selbst im antiken Nahen Osten, Griechenland und Rom finden ${ }^{28}$ und bestimmte Formen des Handels in prähistorischen Gesellschaften können ebenfalls als frühe Methoden einer Versicherung begriffen werden. ${ }^{29}$

Die Originalität des Renaissancekonzepts des Risikos ist die spezifische Kombination einer offenen Zukunft mit Rationalität. Das entscheidende Moment dieses Konzepts ist, dass das Risiko berechnet werden kann. Die Grundlage der Risikoberechnung war die Entdeckung mathematischer Wahrscheinlichkeiten. Die ersten systematischen Studien wurden von Girolamo Cardano im 16. Jahrhundert vorgenommen, der die exakte mathematische Wahrscheinlichkeit eines summenmäßig bestimmten Wurfergebnisses bei der Verwendung von zwei Würfeln erstmals berechnete. ${ }^{30}$ Dieser erste Ansatz wurde über die nächsten hundert Jahre weiter entwickelt zum Konzept des (mathematischen) Erwartungswerts und namentlich Christiaan Huygens war hier maßgebend, der das erwartete Ergebnis eines Spiels berechnete, welches dem gewichteten Durchschnitt aller möglichen Ergebnisse entspricht. ${ }^{31}$

Im 18. Jahrhundert kam dann ein Mathematiker der Bernoulli-Familie zu dem Schluss, dass der Preis, den man bereit war, im Markt zu bezahlen, nicht der (objektive) Erwartungswert war, sondern vielmehr dem erwarteten (subjektiven) Nutzen entsprach und dass Individuen einen zunehmend kleineren Nutzen aus jeder zusätzlichen Währungseinheit gewinnen. ${ }^{32}$ Dieser abnehmende Grenznutzen an Wohlfahrt führt zu dem psychologischen und ökonomischen Phänomen der Risikoaversion. ${ }^{33}$ Darüber hinaus bewerten Individuen Verluste höher als Gewinne gleicher

28 C.F. Trennery, The Origin and Early History of Insurance, London: P. S. King \& Son 1926, S. 4 ff.; zum mittelalterlichen Verständnis im Rahmen der Lehre über den Wucher vgl. B. P. Priddat, Zufall, Schicksal, Irrtum, Marburg 1993, S. 25 ff.

29 Vgl. R. A. Posner, A Theory of Primitive Society with special Reference to Law, Journal of Law and Economics 23 (1980), S. 1-53; zum Risiko als Produktionsfaktor H.-W. Sinn, Gedanken zur volkswirtschaftlichen Bedeutung des Versicherungswesens, Zeitschrift für das gesamte Versicherungswesen 77 (1988), S. 1-27 (13 ff.).

30 Vgl. P. L. Bernstein, Against the Gods: The Remarkable Story of Risk, New York: John Wiley and Sons 1996, S. 47 ff.; L. E. Maistrov, Probability Theory, A Historical Sketch, New York: Samuel Kotz Academic Press 1974, S. 18 ff.; Franklin, Science (Fn. 27), S. 296 ff.

31 Vgl. I. Hacking, The Emergence of Probability: A Philosophical Study of Early Ideas about Probability, Induction and Statistical Inference, Cambridge: Cambridge University Press 1975, S. 92 ff., der die Zeit um 1660 als „Geburtsstunde der Wahrscheinlichkeit“ ansieht (S. 11); s.a. Maistrov, Theory (Fn. 30), S. 48 ff.; zu der komplizierten und verschlungenen Beziehung zwischen mathematischer Wahrscheinlichkeit, statistischen Daten und Versicherungen s. ferner L. J. Daston, The Domestication of Risk: Mathematical Probability and Insurance 1650-1830, in: L. Krüger et al. (Hrsg.), The Probabilistic Revolution, Vol. 1: Ideas in History, Cambridge, Mass.: MIT Press 1987, S. 237-260.

32 D. Bernoulli, Specimen theoriae novae de mensura sortis, in: Commentarii academiae scientiarum imperialis Petropolitanae 6 (1738), S. 175-192; deutsche Übersetzung in: A. Pringsheim, Die Grundlage der Modernen Wertlehre, Berlin 1896, S. 21-60.

33 Vgl. z.B. M. Friedman/L. J. Savage, The Utility Analysis of Choices Involving Risk, Journal of Political Economy 56 (1948), 279-304; K. J. Arrow, The Theory of Risk Aversion (1965), in: ders., Essays in the Theory of Risk-Bearing, Amsterdam: North-Holland Pub. Co. 1971, S. 90-120; kritisch M. Rabin/ R. H. Thaler, Anomalies. Risk Aversion, Journal of Economic Perspectives 15 (2001), S. 219-232; s. ferner u. Fn. 56. 
Größenordnung. Dieses Phänomen der Verlustaversion begünstigt Versicherungen im Allgemeinen, da die Versicherungsnehmer bereit sind, mehr zu zahlen als den zu erwartenden Wert des möglichen Schadens. ${ }^{34}$

Diese Haltung der Menschen gegenüber Risiken ist ebenso durch die Tatsache charakterisiert, dass die Akzeptanz von Risiken generell sehr viel höher ist, wenn das Risiko freiwillig übernommen wird, als wenn das Risiko (unfreiwillig) durch eine dritte Seite aufgezwungen wird. ${ }^{35}$ Allerdings sollte diese Differenzierung nicht zur Grundlage einer kategorialen Unterscheidung zwischen Gefahr und Risiko gemacht werden, wie es von Niklas Luhmann vorgeschlagen worden ist, der Gefahr als aufgezwungen und Risiko als auf menschlicher Entscheidung beruhend definiert. ${ }^{36}$

\section{B. Methoden der Risikosteuerung}

Da die Welt voller Risiken ist, hat die Menschheit immer versucht, mit den erkannten Risiken umzugehen. Grundsätzlich lassen sich drei verschiedene Methoden unterscheiden, Risiken allgemein zu beeinflussen, sie zu modifizieren und zu mildern. ${ }^{37}$

\section{Risikoreduzierung}

Die verbreiteste und auch vorzugwürdige Methode ist die generelle Vermeidung und Reduzierung von Risiken. Menschen versuchen, Risiken zu minimieren, indem sie die erwarteten Risiken entweder ganz ausschalten oder jedenfalls substantiell reduzieren. Dieser Ansatz dominiert vor allem das Öffentliche Recht. Die Abwehr konkreter Gefahren ist das klassische Modell der Risikoreduzierung. Sicherheitsregulierungen sind das primäre Beispiel dieser Strategie. Das Strafrecht lässt sich wahrscheinlich als die älteste Methode der Risikoreduzierung durch Regelung verstehen. ${ }^{38}$ Diese Konzentration und fast ausschließliche Fokussierung auf eine Risikoreduzierung übersieht jedoch, dass Risiken und Verluste häufig unvermeidlich sind. In diesen Fällen versagt das Konzept der Risikosteuerung durch Risikoreduktion in erheblichem Umfang, wenn auch nicht völlig. Allerdings existieren zwei weitere Methoden der Risikosteuerung, die hier ansetzen und häufig übersehen werden.

\section{Risikoverlagerung}

In vielen Fällen kann es außerordentlich sinnvoll sein, Risiken neu zuzuweisen. Grundsätzlich gibt es zwei Methoden der Risikoreallokation, die sich gegenseitig

34 Zur Verlustaversion s. Fn. 61.

35 Grundlegender Artikel: C. Starr, Social Benefit versus Technological Risk, Science 165 (1969), S. $1232 \mathrm{ff}$.

36 N. Luhmann, Soziologie des Risikos, Berlin/New York 1991, S. $30 \mathrm{f.}$

37 Vgl. zu dieser Differenzierung grundlegend Moss, All (Fn. 26), S. $17 \mathrm{ff}$.

38 Vgl. P. O'Malley, The Government of Risks, in: The Blackwell Companion to Law and Society, 2007, S. 292-308 (295 ff.); H. Schmidt-Semisch, Kriminalität als Risiko. Schadenmanagement zwischen Strafrecht und Versicherung, München 2002, S. 19 ff., 109 ff. 
ergänzen. So ist es möglich, schlicht und einfach Risiken zu verlagern oder das Risiko auf breitere Schultern zu verteilen. ${ }^{39}$ Ein klassisches Beispiel für die Verlagerung von Risiken sind Haftungsregeln. So kann z.B. die Haftung für eventuelle Schäden und Risiken vom Verkäufer zum Käufer verlagert werden. ${ }^{40}$ Diese Verlagerung kann dabei einerseits durch einen privatrechtlichen Vertrag zwischen Markteilnehmern oder andererseits durch staatliche Gesetze vorgenommen werden. Der zugrundeliegende Zweck einer solchen Regelung kann sowohl auf moralischen Argumenten im Hinblick auf die Verantwortlichkeit für den Schaden beruhen als auch letztlich dazu dienen, den mächtigeren oder finanzkräftigeren Partner bzw. die Partei infolge der Überbürdung der Risikolast zu veranlassen, das Risiko zu reduzieren. Eine andere Methode der Risikoverlagerung, die in der letzten großen Finanzmarktkrise eine prominente Bedeutung erlangt hat, sind die sogenannten Futures und Derivate. Sie verlagern und diversifizieren das Risiko in der Zeit sowie zu weniger risiko-aversen Investoren. ${ }^{41}$

\section{Risikoverteilung}

Schließlich ist es möglich, Risiken zu verteilen. Das ist besonders sinnvoll, wenn die Risiken zumindest statistisch unvermeidlich sind. In diesem Fall ist das Risiko im Allgemeinen gut bekannt und kann durch alle möglichen Formen von Versicherungen sowie durch eine entsprechende Portfolioauswahl diversifiziert und verteilt werden. ${ }^{42}$ Aktien sind eine weitere Form der Verteilung von Risiken und Gewinnen. Das entscheidende definitorische Element ist, dass die Risikoverteilung in der Regel lediglich das individuelle Risiko nicht aber das aggregierte oder totale Gesamtrisiko reduziert. ${ }^{43}$ Die Strategie der Risikoverteilung zwischen verschiedenen Personen ist das Grundprinzip aller Versicherungen seit den Renaissance-Seeversicherungen. Heutzutage sind Versicherungen eine universale und allgemein verbreitete Form der Risikosteuerung, die jedermann vertraut ist, beginnend mit der Auto- und Feuerversicherung bis hin zu den verschiedenen Arten der Kranken-, Invaliditäts- oder Le-

39 Terminologie von Moss, All (Fn. 26), S. 17 ff. (shift or spread risks).

40 S. S. Shavell, Liability for Harm versus Regulation of Safety, Journal of Legal Studies 13 (1984), S. 357-374; vgl. auch F. Dietz/U. di Fabio/K. Vieweg, Technische Risiken und Gefährdungshaftung, Köln et al. 2006, S. 23 ff., 30 ff. zur Gefährdungshaftung als Alternative zur ordnungsrechtlichen Risikosteuerung; zur unterschiedlichen Wirkung verschuldensunabhängiger oder verschuldensabhängiger Haftung sowie Versicherungen auf die Risikoallokation s. S. Shavell, On Liability and Insurance, Bell Journal of Economics 13 (1982) S. 120-132.

41 Vgl. P. H. Huang, A Normative Analysis of New Financially Engineered Derivatives, Southern California Law Review 73 (2000), S. 471-521.

42 Die klassische Studie ist H. M. Markovitz, Portfolio Selection: Efficient Diversification of Investments, New York: John Wiley \& Sons 1959; diese Methode kann von jedem Individuum für sich selbst angewendet werden, wohingegen Versicherungen Risiken interpersonell verteilen.

43 Vgl. Moss, All (Fn. 26), S. 29 ff.; das Gesetz der großen Zahl kann allerdings bewirken, daß durch die Bündelung der Risiken (pooling of risks) auch das totale Risiko reduziert wird, s. M. V. Pauly, The Economics of Moral Hazard, American Economic Review 58 (1968), S. 531-537 (532). 
bensversicherungen. ${ }^{44}$ In der Regel ist diese Form der Risikoverteilung dem Markt und seinen Teilnehmern vorbehalten, die eine Vielfalt von Versicherungen anbieten, selbst eine Versicherung gegen eine Invasion vom Mars. Allerdings stellt nicht selten der Staat Versicherungen bereit, wofür die Sozialversicherungen von Otto von Bismarck in den 1880er Jahren das erste und bekannteste Beispiel darstellen. ${ }^{45}$ Ebenso bekannt sind die Maßnahmen zur Gewährleistung sozialer Sicherheit im New Deal in den Vereinigten Staaten von Amerika, ${ }^{46}$ die seit der Präsidentschaft Ronald Reagans zunehmend in Frage gestellt werden.

\section{Probleme der Risikosteuerung durch den privaten Sektor}

In einem freien demokratischen Verfassungsstaat mit einer Marktwirtschaft fällt die Risikosteuerung zunächst in die Verantwortung des Einzelnen und des Markts. Das betrifft alle drei bereits erwähnten Methoden der Risikosteuerung in gleicher Weise. In der Regel wird der Einzelne versuchen, zunächst selbst Risiken soweit wie möglich zu reduzieren. Man wird sich um seine eigene Gesundheit bemühen, Unfälle im Straßenverkehr ebenso wie finanzielle Risiken zu vermeiden suchen, soweit dies möglich und nützlich ist. Ebenso ist die Risikoverlagerung ein essentielles Element privater Verträge. Versicherungen werden ebenfalls meistens vom Markt bereitgestellt. ${ }^{47} \mathrm{Al}-$ lerdings gibt es Grenzen für die private Risikosteuerung, die auf mehreren Problemen beruhen, die spezifisch risikobezogen sind.

\section{Asymmetrische Information}

Einige risikobezogene Formen des Marktversagens sind seit dem 19. Jahrhundert wohl bekannt und basieren auf der Tatsache asymmetrischer Information. ${ }^{48}$ Das Problem nachteiliger Auswahl (adverse selection) wurde ausdrücklich identifiziert als Begriff und Phänomen auf dem Gebiet der Lebensversicherungen. ${ }^{49}$ Adverse Selektion liegt vor, wenn einzelne Personen mehr über ihre eigenen Risiken wissen als ihre Versicherungen. Während z.B. eine Person mit einer tödlichen Krankheit, die ausschließlich ihr selbst bekannt ist, eine hohe Lebensversicherung erwerben mag,

44 Vgl. auch H.-B. Schäfer/C. Ott, Lehrbuch der ökonomischen Analyse des Zivilrechts, 4. Aufl., Berlin et al. 2005, S. $134 \mathrm{ff}$.

45 Zur Einführung M. Stolleis, Geschichte des Sozialrechts in Deutschland, Stuttgart 2003, S. 52 ff.

46 Vgl. Moss, All (Fn. 26), S. $180 \mathrm{ff.}$

47 Der Grund für ökonomisch handelnde Individuen, Risiken zu kaufen und zu verkaufen ist nicht nur Diversifikation, sondern auch die Tatsache, daß Menschen unterschiedliche Einstellungen gegenüber Risiken haben, daß einige stärker risikoavers sind als andere; weitere Gründe sind unterschiedliche Risikobewertung und Portfolio-Diversifikation. Vgl. Moss, All (Fn. 26), S. 34 f.

48 Zum Problem der asymmetrischen Information am Beispiel des Gebrauchtwagenmarktes s. allgemein G. A. Akerlof, The Markets for Lemons: Quality, Uncertainty and the Market System, Quarterly Journal of Economics 84 (1970), S. $488 \mathrm{ff}$.

49 Moss, All (Fn. 26), S. 36. 
kann eine Person mit guten Risiken ${ }^{50}$ die Versicherung verlassen und dadurch Geld sparen, indem sie in anderer Weise ihr Geld investiert. Dem kann bis zu einem gewissen Grad durch Gesundheitsuntersuchungen und -überprüfungen begegnet werden. Jedoch können diese entweder schwierig, teuer oder in bestimmten Fällen unmöglich sein. ${ }^{51}$

In der neueren Diskussion ist das Problem des sogenannten moral hazard noch bekannter, das in verschiedenen Kontexten auftritt. Es wurde erstmals entdeckt im Bereich der Feuerversicherung, wenn Versicherungsnehmer entweder Brandstiftung begehen oder sich des Betrugs, vorsätzlicher Unvorsichtigkeit oder Nachlässigkeit bei Eintritt des Versicherungsfalls schuldig machen. ${ }^{52}$ Moral hazard kann definiert werden durch den Anreiz zu einem Verhalten, das die Schadenswahrscheinlichkeit einer Situation oder einer Aktivität erhöht, wobei derjenige, der die Schadensneigung steigert, das Geschehen bzw. den Eintritt des Schadens maßgebend beeinflussen kann, die Schadensfolgen aber einem Dritten oder der Allgemeinheit zur Last fallen. ${ }^{53}$ M.a.W.: Derjenige, der das Risiko steigert, ist von den Schadensfolgen in jedem Fall entlastet, so dass die gewöhnliche Risikoaversion reduziert oder beseitigt wird. Das ist zum Teil mit asymmetrischer Information nach Vertragsschluss verbunden, diese ist aber kein notwendiges Element für moral hazard. Moral hazard ist in erster Linie ein Versicherungsproblem, betrifft aber ebenso jede Form des (erzwungenen) bail-out und ist damit nicht auf Vertragsverhältnisse beschränkt. Obwohl dieses Phänomen bereits in den 1860er Jahren erkannt worden ist, findet sich die erste formalisierte und systematische Behandlung in der ökonomischen Wissenschaft erst etwa hundert Jahre später. ${ }^{54}$

50 Der Begriff der guten Risiken (good risks) hat sich auch im deutschen Schrifttum eingebürgert, weil damit nicht lediglich geringe (Schadens)Risiken erfaßt werden. Das Phänomen der adverse selection ist auch nicht auf das Stadium vor Vertragsabschluß beschränkt, sondern gilt gerade auch für den Fall der Beendigung des Vertrags.

51 Vgl. M. Rothschild/J. Stieglitz, Equilibrium in Competitive Insurance Markets: An Essay on the Economics of Imperfect Information, Quarterly Journal of Economics 90 (1976), S. 629-649; H. Gravelle, Insurance Law and Adverse Selection, International Review of Law and Economics 11 (1991), S. 23-45, bes. S. 24 f.; zum Problem der Gentests in diesem Kontext vgl. D. J. Christiansen, Genetic Testing: Risk Classification and Adverse Selection, Journal of Insurance Regulation 15 (1996), S. 75-79.

52 Vgl. T. Baker, On the Genealogy of Moral Hazard, Texas Law Review 75 (1996), S. 237-292 (248 f.); zu noch früheren Beobachtungen vgl. Moss, All (Fn. 26), S. 38.

53 Moral hazard ist nicht allein ein Problem der Informationsasymmetrie, sondern auch Ergebnis der Tatsache, daß das Verhalten nicht (immer) beobachtbar ist, s. B. Hallström, Moral Hazard and Observability, Bell Journal of Economics 10 (1979), S. 74-91, und deswegen oder aus anderen Gründen von demjenigen, der den Schaden tragen muß, nicht verhindert werden kann; zur Währungsunion als eine Art Versicherung mit der Folge von moral hazard und adverse selection s. W. Schelkle, The Political Economy of Fiscal Policy Coordination, in: EMU: From Disciplinarian Device to Insurance Arrangement, Journal of Common Market Studies 43 (2005), S. 371-91 (377ff., bes. 384 f.).

54 K. J. Arrow, Uncertainty and the Welfare Economics of Medical Care, American Economic Review 53 (1963), S. 941-973, auch in: ders., Essays (Fn. 33), S. 177-211. 
Es gibt aber weitere Informationsprobleme, die nicht auf asymmetrischer Information beruhen, aber nichtsdestoweniger Marktversagen verursachen. Das betrifft vor allem die Fälle, in denen keine der beteiligten Parteien oder überhaupt niemand hinreichende Informationen über die fraglichen Risiken erlangen kann, weshalb diese in keiner Weise berechenbar sind. Dieses Phänomen gilt vor allem für Katastrophen und Unglücksfälle, die nicht vorhersehbar sind und zugleich extrem hohe Kosten verursachen. In diesen Fällen wird häufig niemand gewillt sein, das Risiko im Markt $\mathrm{zu}$ versichern, weil die Informationen entweder nicht existieren oder es zu teuer ist, sie zu erlangen. Lloyds versichert fast alles, aber nicht gegen einen nuklearen Winter.

\section{Risikowahrnehmung}

Ein relativ neues Feld der Forschung, das lange von den ökonomischen Wissenschaften vernachlässigt worden ist, sind die sogenannten Wahrnehmungsprobleme, welche die ökonomischen Annahmen der Rationalität und Konsistenz unterminieren. Die Grundlagen wurden bereits in den 1920er Jahren von Frank Knight gelegt, der die grundlegende Unterscheidung zwischen Risiko und Ungewissheit in die wissenschaftliche Diskussion eingeführt hat. Während danach Risiko messbare Wahrscheinlichkeiten betrifft, bezieht sich Ungewissheit auf nicht berechenbare und vielleicht sogar unbekannte Wahrscheinlichkeiten. ${ }^{55}$ Diese Unterscheidung wurde allerdings lange Zeit von den Ökonomen mit der Begründung, dass präzise Wahrscheinlichkeiten für die Erwartungsnutzentheorie nicht notwendig seien, als irrelevant betrachtet. Es wurde angenommen, dass es ausreichend sei, die Wahrscheinlichkeit auf subjektive Schätzungen zu gründen, die als objektive Fakten behandelt werden konnten. ${ }^{56}$ Die Theorie eines subjektiven erwarteten Nutzens konnte selbst außer Acht lassen, wenn sich Leute in vollständiger Unkenntnis über die objektiv existierenden Wahrscheinlichkeiten befanden. Diese Theorie wurde allerdings erschüttert durch die Entdeckung des sogenannten Ellsberg-Paradoxes, das aufzeigt, dass sogar die aktuelle Entscheidung ausgebildeter Ökonomen die Konsistenzannahme dieser sub-

55 F. H. Knight, Risk, Uncertainty, and Profit Boston: Hart, Schaffner \& Marx 1921, Nachdr. 1971, S. 197 ff., insbes. 233, s.a. 19 f.; heute wird teilweise zwischen drei Stufen der Unsicherheit differenziert: 1 . bekannte, statistisch berechenbare Wahrscheinlichkeiten, 2. unsichere Wahrscheinlichkeiten bekannter Ereignisse, 3. auch die möglichen Ereignisse sind unbekannt. Die beiden letzten Konstellationen sind Fälle der Ungewißheit i.S.v. Knight.

56 M. Friedman/L. J. Savage, The Expected Utility Hypothesis and the Measurability of Utility, Journal of Political Economy 60 (1952), S. 463-474; L. J. Savage, The Foundations of Statistics, 1954; der Ansatz basiert auf F. R. Ramsey, The Foundations of Mathematics, 1931, und J. von Neumann/O. Morgenstern, Theory of Games and Economic Behavior, Princeton: Princeton University Press 1944 (3. Aufl. 1953); s. aber auch bereits die klassische Darstellung von A. Marshall, Principles of Economics, 8. Aufl., London: Macmillan 1920 (neu gesetzt 1949), S. 460 f.; zu diesem Ansatz s. jetzt auch J. Hirschleifer/J. G. Riley, The Analytics of Uncertainty and Information, 1992, S. 7 ff., die folgerichtig jeden Unterschied zwischen Risiko und Unsicherheit negieren; vgl. ferner P. J. Neumann/P. E. Politser, Risk and Optimality, in: Yates, Risk-Taking Behavior (Fn. 25), S. 27-47 (30 ff.); einführend J. Schumann/U. Meyer/W. Ströbele, Grundzüge der mikroökonomischen Theorie, 8. Aufl., Berlin et al. 2007, S. $427 \mathrm{ff}$. 
jektiven Theorie verletzte. ${ }^{57}$ Seitdem sind alle möglichen Arten und Formen irrationalen Verhaltens im Hinblick auf die Wahrnehmung in Entscheidungsprozessen entdeckt worden. ${ }^{58}$ Insbesondere Techniken der Einschätzung von Risiken durch Individuen sind in mehrerlei Hinsicht voreingenommen und vorurteilsbeladen. So zeigte bereits das Ellsberg-Paradox, dass Menschen versuchen, unklare oder unbekannte Wahrscheinlichkeiten sogar um den Preis der Inkonsistenz zu vermeiden. Die Menschen sind nicht nur risikoavers, sondern auch ambiguitätsavers, d.h. sie suchen ebenso Unklarheit und Unsicherheit zu vermeiden. ${ }^{59}$

Weiterhin verwenden Individuen meist heuristische Techniken, um Wahrscheinlichkeiten zu schätzen, die jedoch auf systematischen Vorurteilen bzw. Fehlern beruhen, was mit dem englischen Begriff des bias prägnant umschrieben wird. ${ }^{60}$ Vier Probleme sind besonders auffallend und verbreitet. $\mathrm{Zu}$ allererst verhalten sich Menschen unterschiedlich, wenn sie mit demselben Risiko konfrontiert sind: Sie übernehmen nur geringere Risiken, wenn die Wahl zwischen verschiedenen Risiken in der Terminologie von Gewinnen formuliert ist und größere Risiken, wenn die Wahl begrifflich als Verlust formuliert ist, weil Verluste als schädlicher angesehen werden. ${ }^{61}$ Außerdem wird allgemein möglichst vermieden, dass bereits getätigte Ausgaben nachträglich sinnlos werden. ${ }^{62}$ Zweitens neigen Menschen dazu, zu großes Gewicht auf die

57 D. Ellsberg, Risk, Ambiguity, and the Savage Axioms, Quarterly Journal of Economics 75 (1961), S. 643-669; W. Fellner, Distortion of Subjective Probabilities as a Reaction to Uncertainty, Quarterly Journal of Economics 75 (1961), S. 670-689; vgl. dazu aus klassischer Sicht H.-W. Sinn, Ökonomische Entscheidungen bei Ungewißheit, Tübingen 1980, S. $24 \mathrm{ff}$.

58 Vgl. aus empirischer Sicht P. J. H. Schoemaker, The Expected Utility Model: Its Variants, Purpose, Evidence and Limitations, Journal of Economic Literature 20 (1982), S. 529-563, bes. 541-552; generelle Kritik bei J. Beckert, What is Sociological about Economic Sociology? Uncertainty and the Embeddedness of Economic Action, Theory and Society 25 (1996), S. 802-840.

59 Ellsberg, Risk (Fn. 57), S. 659-669; W. K. Viscusi, Rational Risk Policy, Oxford_Oxford University Press 1998, S. 17 ff. zur risk ambiguity aversion.

60 Grundlegender Artikel von A. Tversky/D. Kahneman, Judgment under Uncertainty, Heuristics and Biases, Science 185 (1974), S. 1124-1131; gute kurze Übersicht D. Kahneman/M. W. Riepe, Aspects of Investor Psychology: Beliefs, Preferences and Biases Investment Advisors Should Know About, Journal of Portfolio Management 24 (1998), 52-65; für das Folgende vgl. die Essaysammlungen: $D$. Kahneman/P. Slovic/A. Tversky (Hrsg.), Judgment under Uncertainty: Heuristics and Biases, Cambridge, UK: Cambridge University Press 1982; und die Fortsetzung D. Kahneman/A. Tversky (eds.), Choices, Values, and Frames, Cambridge, UK: Cambridge University Press 2000; P. Slovic (Hrsg.), The Perception of Risk, London: Earthscan 2000; T. Gilovich/D. Griffin/D. Kahneman (Hrsg.), Heuristics and Biases, Cambridge: Cambridge University Press 2002; s.a. das einflußreiche Konzept der beschränkten Rationalität bei H. A. Simon, Models of Man. Social and Rational, New York: Wiley 1957, S. 196 ff.; ders, Administrative Behavior, 3. Aufl., New York: Free Press 1976, S. 80 ff.

61 A. Tversky/D. Kahneman, The Framing of Decisions and the Psychology of Choice, Science 211 (1981), S. 453-458, auch in: Judgment (Fn. 60), S. 3-20; zur Verlust-Aversion selbst (Menschen gewichten Verluste stärker als gleich große Gewinne) s. A. Tversky/D. Kahneman, Prospect Theory: An Analysis of Decision under Risk, Econometrica 47 (1979), S. 263-291, auch in: Choices (Fn. 60), S. 17-43, und von den gleichen Autoren, Advances in Prospect Theory, Journal of Risk and Uncertainty 5 (1992), S. 297-323, auch in: Choices (Fn. 60), S. 44-65; vgl. auch den sog. endowment effect; dazu $R$. Thaler, Toward a Positive Theory of Consumer Choice, Journal of Economic Behavior and Organization 1 (1980), S. 39-60 (44 f.).

62 Zum Problem der sunk costs s. Thaler, Theory (Fn. 61), S. 47 ff. 
besonders gut verfügbaren und erinnerlichen Informationen bezüglich des relevanten Problems zu legen. Während in manchen Fällen die Verfügbarkeit der Information als Anhaltspunkt für die Häufigkeit eines Schadens durchaus nützlich sein kann, verführt dieses Phänomen Menschen häufig dazu, sich auf erinnerliche aber nicht repräsentative Informationen zu stützen. Ein Autounfall auf der Straße, den man gesehen hat, erhöht etwa temporär die subjektive Wahrscheinlichkeit eines Autounfalls im Allgemeinen deutlich. ${ }^{63}$ Neu entdeckte Risiken ziehen eine überzogene Aufmerksamkeit auf sich. ${ }^{64}$ Generell führt Publizität zur Überschätzung von Risiken. ${ }^{65}$ Drittens hat die Forschung ebenso ein übermäßiges Vertrauen und ein OptimismusVorurteil hinsichtlich der Einschätzung persönlicher Risiken beobachtet. ${ }^{66}$ Beispielsweise glaubt die große Mehrheit der Autofahrer, dass sie ein geringeres Risiko laufen, in einen Unfall verwickelt zu werden, als der Durchschnitt. ${ }^{67}$ Ähnliche Beobachtungen sind für Arbeitsunfälle oder das persönliche Risiko, von Arbeitslosigkeit betroffen zu sein, gemacht worden. ${ }^{68}$ In diesem Kontext führen zwei weitere Phänomene zu Fehleinschätzungen. Generell schätzen die Einzelnen die Wahrscheinlichkeit persönlicher Betroffenheit durch negative Ereignisse geringer, und durch positive Ereignisse höher als durchschnittlich ein und zugleich verzerrt die Kontrollierbarkeit der Ereignisse durch den Betroffenen das Wahrscheinlichkeitsurteil zusätzlich. ${ }^{69}$ Schließlich werden viertens extreme Wahrscheinlichkeiten gewöhnlich grob falsch eingeschätzt. In hohem Maße unwahrscheinliche Ereignisse werden entweder völlig außer Acht gelassen oder überbewertet, und die Differenz zwischen einer hohen Wahrscheinlichkeit und Gewissheit wird entweder vernachlässigt oder übertrieben. ${ }^{70}$ Ereignisse von extrem niedriger Wahrscheinlichkeit aber mit sehr hohen Schadensfolgen, wie Naturkatastrophen ${ }^{71}$ werden daher entweder gar nicht versichert

63 Tversky/Kahneman, Judgment (Fn. 60), S. 1127; vgl. dazu verschiedene allgemeine Essays in: Kahneman et al., Judgment (Fn. 60), S. 23 ff.; Gilovich et al., Heuristics (Fn. 60), S. 19 ff.

64 Viscusi, Risk Policy (Fn. 59), S.17.

65 Viscusi, Risk Policy (Fn. 59), S. $21 \mathrm{ff}$.

66 Vgl. diverse Artikel in: Kahneman et al., Judgment (Fn. 60), S. 287 ff.; ebenso die Artikel in: Gilovich et al., Heuristics (Fn. 60), S. 313 ff.; kurz Kahneman/Riepe, Aspects (Fn. 60) S. 53 f.

67 O. Svenson, Are We All Less Risky and More Skillful Than Our Fellow Drivers?, Acta Psychologica 47 (1981), S. 143-148.

68 N. D. Weinstein, Optimistic Biases about Personal Risks, Science 246 (1989), S. 1232 f.; ders., Why it Won't Happen to Me: Perception of Risk Factors and Susceptibility, Health Psychology 3 (1984), S. 431-457; s.a. W. Kip Viscusi, The Value of Risks to Life and Health, Journal of Economic Literature 31 (1993), S. 1912-1946; ders., Fatal Tradeoffs, 1992, S. 34-50, 51-74.

69 N. D. Weinstein, Unrealistic Optimism about Future Events, Journal of Personality and Social Psychology 39 (1980), S. 806-820; D. Zakay, The Influence of perceived Event's Controllability on its Subjective Occurrence Probability, Psychological Record 34 (1984), S. 232-240.

70 Kahneman/Tversky, Prospect Theory (Fn. 61), S. 283.

71 Vgl. P. Slovic/H. Kunreuther/G. F. White, Decision Processes, Rationality and Adjustment to Natural Hazards, in: Slovic, Perception (Fn. 60), S. 1-32, s. auch die vier Artikel von P. Slovic/B. Fischhoffl S. Lichtenstein, ebd., S. 32-50, 104-120, 121-136, 137-153. 
oder gewaltig überversichert. ${ }^{72}$ In dieselbe Richtung weist auch die Tatsache, dass Menschen einen völlig unverhältnismäßigen Preis zu entrichten bereit sind für die völlige Ausschaltung von Risiken. ${ }^{73}$ Außerdem scheinen in der Rückschau vergangene Ereignisse unvermeidlich, so dass die Menschen zu dem Glauben neigen, dass diese Unvermeidlichkeit auch schon im Vorhinein offensichtlich war. ${ }^{74}$ Austauschbeziehungen des privaten Marktes sind daher in vielen Fällen nicht ökonomisch optimal. ${ }^{75}$ Zudem konzentrieren sich Individuen auf unterschiedliche Risiken, selektieren Risiken und sind über die gewünschten Aussichten und Ziele sowie Bewertungen völlig uneinig. ${ }^{76}$ Dementsprechend gehen Menschen Risiken in höchst unterschiedlichem Maß ein. ${ }^{77}$ Aus diesem Grund ist sowohl die Risikowahrnehmung als auch die Risikosteuerung sehr stark von kulturellen Haltungen und Vorlieben beeinflusst. Entscheidungen der Risikosteuerung sind folglich sehr oft politischer Natur. $^{78}$

\section{Verpflichtungsprobleme}

Eine weitere Kategorie des Marktversagens ist eine Konsequenz aus staatlichen Reaktionen auf bestimmte Risiken und resultiert aus der spezifischen Unfähigkeit der privaten Akteure definitive Verpflichtungen für die Zukunft einzugehen. Das Phänomen ist daher gelegentlich als Verpflichtungsproblem (commitment problem) bezeichnet worden. ${ }^{79}$ Dem liegt Folgendes zugrunde: Die Verluste, die durch große Naturkatastrophen oder andere Formen von Unglücksfällen verursacht werden und eine große Anzahl von Bürgern betreffen, zwingen demokratische Regierungen regelmäßig dazu, die Opfer zu unterstützen und gegebenenfalls einen finanziellen Ausgleich zu gewähren. Zugleich wird das Wissen darum, daß die Regierung entsprechend handeln wird, die Menschen davon abhalten, sich gegen solche Risiken zu

72 Vgl. H. Kunreuther/R. Ginsberg/L. Miller et al., Disaster Insurance Protection: Public Policy Lessons, New York: Wiley 1978.

73 Viscusi, Risk Policy (Fn. 59), S. 15, 99; $90 \%$ der Kosten fallen für die Beseitigung der letzten $10 \%$ des Risikos an.

74 B. Fischhoff, Hindsight - Foresight: The Effect of Outcome Knowledge on Judgment Under Uncertainty, Journal of Experimental Psychology: Human Perception and Performance 1 (1975), S. 288-299.

75 Vgl. hierzu allgemein K.. Arrow, Risk Perception in Psychology and Economics, Economic Inquiry 20 (1982), S. 1-9.

76 Zum Problem der Bewertung und der Einschätzung der Tatsachen B. Fischhoff et al., Acceptable Risk, New York: Cambridge University Press 1981, S. 15 f f., $22 \mathrm{f} \mathrm{f.} 43 \mathrm{ff}$.

77 P. Bromiley/S. P. Curley, Individual Differences in Risk Taking, in: Yates, Risk-Taking Behavior (Fn. 25), S. 87-132.

78 See M. Douglas/A. Wildavsky, Risk and Culture, Berkeley: UCP 1982, die Risiko als ein Produkt von Wissen und Konsens über die am häufigsten gewünschten Aussichten und Ziele definieren (S. 5).

79 Vgl. Moss, All (Fn. 26), S. 45 ff. 
versichern. Das begründet „ein Dilemma für staatliche Reaktionen“ (,, dilemma of government responsiveness“). ${ }^{80}$

Es stellt ferner ein implizites Marktversagen dar, dass Individuen einer Generation nicht mit denen einer anderen (noch gar nicht geborenen) Generation in geschäftlichen Austausch treten können. Es gibt keine bindende Verpflichtung zukünftiger Generationen durch gegenwärtige Marktteilnehmer. ${ }^{81}$ Das betrifft insbesondere sogenannte systematische Risiken, die durch die Tatsache definiert sind, dass sie nicht zu irgendeinem Zeitpunkt im Zeitablauf diversifiziert werden können. Ein Risiko, das praktisch jedermann zu demselben Zeitpunkt trifft und berührt, kann überhaupt nicht verteilt werden. ${ }^{82}$ Deshalb ist ein massiver Nuklearkrieg ebenso wenig durch den Privatsektor versicherbar wie die negativen Folgen makroökonomischer Konjunkturverläufe. Systematisches Risiko ist zu dem Zeitpunkt, zu dem es eintritt, nicht diversifizierbar, wohl aber über die Zeit, obwohl dies nicht durch private Marktteilnehmer möglich ist. Das ist der Grund dafür, dass in diesen Fällen der Staat einschreiten kann und muss.

Ein anderes Verpflichtungsproblem wird durch Regulierung geschaffen. In allen modernen ökonomischen Systemen ist gesetzlich geregelt, dass jedermann in Konkurs gehen kann und im Fall der Insolvenz einen gewissen Schutz beanspruchen kann. Aus diesem Grund kann sich niemand verpflichten, hinsichtlich seiner zukünftigen Verbindlichkeiten nicht in die Insolvenz zu gehen. Bei den meisten Markttransaktionen ist dieses Problem beherrschbar, obwohl dadurch ein Anreiz geschaffen wird, sich durch die Insolvenz von allen Verpflichtungen zu befreien. Es kann aber ein spezifisches Problem für die individuellen Investitionen in private Pensionsfonds darstellen, da sogar eine besonders niedrige Wahrscheinlichkeit der Insolvenz die Menschen davon abhält, solchen Pensionsfonds ihre gesamten Lebensersparnisse anzuvertrauen. Dieses Faktum stellt daher eine der wesentlichen Rechtfertigungen für öffentlich-rechtliche soziale Sicherungs- bzw. Versicherungssysteme dar. ${ }^{83}$

Dieses Recht auf Insolvenz ist ferner ein zusätzlicher Grund für ein weiteres Verpflichtungsproblem, das als Unveräußerlichkeit von Humankapital bezeichnet wird.

80 D. Rodrik/R. Zeckhauser, The Dilemma of Government Responsiveness, Journal of Policy Analysis and Management 7 (1988), S. 601-620; s.a. S. Coate, Altruism, the Samaritan's Dilemma, and Government Transfer Policy, American Economic Review 85 (1995), S. 46-57.

81 J. E. Stieglitz, On the Relevance or Irrelevance of Public Financial Policy: Indexation, Price Rigidities and Optimal Monetary Policies, in: Rüdiger Dornbusch/Mario Henrique Simonsen (Hrsg.), Inflation, Debt, and Indexation, Cambridge, Mass.: MIT Press 1983, S. 183-222 (186); s.a. P. A. Diamond, A Framework for Social Security Analysis, Journal of Public Economics 8 (1977), S. $275-298$ (279 ff.).

82 Moss, All (Fn. 26), S. 46 f.; systematisches Risiko muß unterschieden werden von systemischem Risiko, bei dem ein ganzes System von dem Versagen einer seiner Einheiten betroffen ist: so wie das Bankensystem vom Konkurs einer Großbank beeinträchtigt sein kann, vgl. M. Hellwig, Risiken im Finanzsektor, Zeitschrift für Wirtschafts- und Sozialwissenschaften, Beiheft 7 (1998), S. 123-151; S. L. Schwarcz, Systemic Risk, Georgetown Law Journal 97 (2008), S. 193-249.

83 Moss, All (Fn. 26), S. 47; see also Diamond, Framework (Fn. 81), S. 289 ff. 
In modernen freien Marktwirtschaften ist das Humankapital unveräußerlich, weil Individuen sich nicht glaubwürdig verpflichten können, ihr zukünftiges Einkommen auf andere zu übertragen, weil sie nicht berechtigt sind auf ihr Recht auf Insolvenz zu verzichten oder ihre Arbeitskraft in die Sklaverei oder Schuldknechtschaft zu verkaufen. Humankapital ist daher ein nicht handelbarer Vermögenswert und Risiken, die das Humankapital betreffen, sind daher nicht diversifizierbar, da sie nicht verkauft oder gekauft werden können. Die Diversifizierung von Humankapitalrisiken erfordert daher ein Eingreifen des Staates durch das Sozialversicherungssystem. ${ }^{84}$

\section{Externalitäten}

Eine weitere Quelle für Risikosteuerungsprobleme durch den privaten Sektor sind die sogenannten Externalitäten. ${ }^{85}$ Externalitäten als solche sind nicht beschränkt auf Risikoprobleme, sondern ein allgemeiner Grund für Marktversagen. ${ }^{86}$ In erheblichem Maß können allerdings Externalitäten auch zu spezifischen Risiken in Beziehung gesetzt werden. In der Risikoterminologie können Externalitäten als quasi automatische Verlagerung von Risiken auf die Allgemeinheit betrachtet werden. In dieser Hinsicht schafft Umweltverschmutzung Risiken für das Gemeinwesen und die Beteiligung des Einzelnen am Straßenverkehr erhöht das Risiko von Schäden für andere Fahrer und Fußgänger. Die Schaffung einer Zwangsversicherung für Autos bedeutet insoweit, dass die Autofahrer gezwungen werden, die Verantwortung für das Risiko zu übernehmen, das sie durch die Nutzung ihres Autos schaffen. ${ }^{87}$ Das wird anschaulich durch die spezifische Form der Autoversicherung in Deutschland.

Risiken können auch dadurch entstehen oder vermehrt werden, dass ein individuelles Verhalten in einem gewissen Maß rational ist, aber durch das Zusammenwirken einer Vielzahl von Einzelentscheidungen auf der Kollektivebene zu irrationalen Ergebnissen führt. Das trifft insbesondere auf konjunkturelle Abschwünge zu, wenn eine sinkende Nachfrage das Angebot reduziert, das seinerseits die Nachfrage reduziert usw. ${ }^{88}$ Im Falle einer Finanzpanik, kann die Furcht vor Verlusten ansteckend wirken und sogenannte bank runs zur Folge haben, die sogar finanziell gesunde Banken in den Ruin treiben können. ${ }^{89}$ Wenn nervöse Anleger ihr Geld vorzeitig ab-

84 R. C. Merton, On the Role of Social Security as a Means for Efficient Risk Sharing in an Economy Where Human Capital is Not Tradeable, NBER Working Paper 743 (Sept. 1981).

85 Davon oder von externen Effekten spricht man, wenn bei der Produktion von Gütern soziale (externe) Kosten entstehen, die der Produzent nicht in seine eigene (interne) Kostenrechnung einstellt, also Dritten oder der Allgemeinheit Kosten auferlegt.

86 Als Einführung vgl. klassisch A. C. Pigou, Wealth and Welfare, London: Macmillan 1912, S. 162-165; ders, The Economics of Welfare, London: Macmillan 1920 (4. Aufl. 1932), S. 131-135, 172-212.

87 Moss, All (Fn. 26), S. 48.

88 Vgl. W. Heun, Staatshaushalt und Staatsleitung, Baden-Baden 1989, S. 124 f.

89 Das Risiko von bank runs liefert die Rechtfertigung für Zentralbanken als Kreditgeber letzter Instanz, s. W. Heun, Die Zentralbank der USA - das Federal Reserve System, Staatswissenschaft und Staatspraxis 9 (1998), S. 241-281 (248f.). 
ziehen, dann legen sie den anderen Anlegern, den Banken und den Investoren Kosten auf und erhöhen das allgemeine Risikoniveau. Dieser Prozess kann sogar zu einem systemischen Risiko für das gesamte Finanzsystem führen, wie man es in der letzten Finanzmarktkrise beobachten konnte. ${ }^{90}$ Der vorzeitige und hastige Abzug von Bankeinlagen kann ebenso als Externalität begriffen werden, die auf die anderen Anleger verlagert wird. Der Hauptaspekt ist jedoch der kollektive Wahn und der Teufelskreis von Rückkopplungen, der die Krise erschwert und die Risiken erhöht. ${ }^{91}$ Diese Situation ist durch den privaten Sektor praktisch nicht beherrschbar und verlangt daher nach einer staatlichen Intervention.

\section{Die Rolle des Staates als ultimativer Risikomanager}

Die Rolle des Staates hängt von dem gewählten ökonomischen System ab. Alle westlichen politischen Systeme haben im Wesentlichen für eine marktwirtschaftliche Ordnung optiert. In einem solchen System hat der Staat spezifische Funktionen, um das Marktversagen zu kompensieren. ${ }^{92}$ Diese Funktionen sind auf verschiedene spezifische Fähigkeiten des Staates gegründet, über die die privaten Marktteilnehmer nicht verfügen. Die Hauptvorzüge des Staates sind seine Möglichkeiten, rechtlich zwingende Regeln zu erlassen und seine Entscheidungen mit legitimer Gewalt durchzusetzen. Daneben treten seine ökonomischen Möglichkeiten der Besteuerung, der Geldschöpfung und als ihre Konsequenz die spezifische Kreditfähigkeit des Staates. Staaten vermögen Kapital in einem solchen Ausmaß zu mobilisieren, wie sie selbst die allerreichsten und mächtigsten Privatrechtssubjekte nicht zu erreichen vermögen. Außerdem verfügt der Staat über einen ausgedehnten bürokratischen Apparat, der spezialisiertes Wissen mit einer hohen Kapazität der Problemlösung jeglicher Art kombiniert. ${ }^{93}$ Diese Fähigkeiten des Staates im allgemeinen sind die Grundlage seiner Rolle als „ultimate risk manager“. 94

Seine klassische Funktion der Risikoreduzierung und Risikovorsorge durch das Recht und seine Durchsetzung ist hauptsächlicher Teil seiner traditionellen Polizeigewalt und durchdringt zahlreiche verschiedene staatliche Aktivitäten. ${ }^{95}$ Die Reduzierung kollektiver Risiken ist darüber hinaus eine natürliche und selbstverständliche Verpflichtung des Staates. Der Staat spielt aber auch die Hauptrolle bei der Verlagerung und Verteilung von Risiken. Seine Regelungs- und Rechtssetzungsgewalt

90 Vgl. W. Heun, Der Staat und die Finanzkrise, JZ 2010, S. 53-62.

91 Moss, All (Fn. 26), S. 49, der auch beobachtet, daß in diesem Fall eine (Einlagen-)Versicherung das Risiko nicht nur verteilen, sondern sogar das Gesamtrisiko reduzieren kann.

92 Klassischer allgemeiner Überblick R. Musgrave/P. B. Musgrave, Public Finance in Theory and Practice, 4. Aufl. New York: McGraw-Hill 1984, S. 7 ff.; s.a. K. Homann/A. Suchanek, Ökonomik, Tübingen 2000, S. $207 \mathrm{ff}$.

93 Vgl. R. Mayntz, Soziologie der öffentlichen Verwaltung, 4. Aufl. Heidelberg 1997, S. 82 ff.

94 So die Formulierung von Moss, All (Fn. 26), S. 49 ff.

95 Vgl. P.-T. Stoll, Sicherheit als Aufgabe von Staat und Gesellschaft, Tübingen 2003, S. 13-263; zum allgemeinen Problem der Risikoregulierung in den USA s. S. Breyer, Breaking the Vicious Circle, 1993. 
kann die gegenwärtigen Bürger wie zukünftige Generationen zwingen, sich an Versicherungssystemen zu beteiligen oder staatliche Versicherungen für die Bürger selbst begründen, einrichten und aufrechterhalten. ${ }^{96}$ Der Staat kann adverse Selektion bekämpfen, indem er eine breite Partizipation erzwingt und jeglichen Austritt von Teilnehmern mit guten Risiken aus den jeweiligen Versicherungssystemen verhindert. Soziale Sicherheit und Versicherungssysteme sowie obligatorische Autoversicherungen sind ein hervorragendes Beispiel für diese Funktion. Der Staat fungiert in einem gewissen Umfang sogar unmittelbar als eine Versicherung gegen hohe Risiken wie Krieg oder natürliche sowie von Menschen verursachte Katastrophen. Dies ist eine Konsequenz seiner ökonomischen Macht und seiner fast unerschöpflichen Vermögensgrundlagen wie auch der Tatsache, dass er bis in die Zukunft hinein besteuern kann. Steuern werden sogar manchmal durch die Idee gerechtfertigt, dass der Staat als Versicherung agiert. ${ }^{97}$ Auch die Möglichkeit von Zentralbanken, als lender of last resort den Geschäftsbanken Finanzmittel zur Verfügung zu stellen sowie ihre Fähigkeit zur Geldschöpfung wirken gewissermaßen als eine Form der Versicherung. Mit seiner polizei- und strafrechtlichen Untersuchungs- und Durchsetzungsmacht kann der Staat den sogenannten moral hazard jedenfalls partiell und besser als private Marktteilnehmer kontrollieren und überwachen, indem er sich zwangsweise Zugriff auf die Informationen der privaten Akteure verschafft. ${ }^{98}$ Fehleinschätzungen von Risiken als Folge von Wahrnehmungsproblemen kann dadurch begegnet werden, dass die betreffenden Informationen zur Verfügung gestellt werden und die Bürger dazu gezwungen werden, die notwendigen Versicherungen zu erwerben, wie das etwa bei der Auto- oder der Feuerversicherung der Fall ist. Die obligatorische Sozialversicherung gegen Arbeitslosigkeit ist ein weiteres prominentes Beispiel für diese Staatsfunktion. Außerdem kann der Staat Externalitäten, die Risiken verursachen, internalisieren, indem er die Verantwortlichen zum Regress verpflichtet oder das betreffende Verhalten oder Vorgehen besteuert oder strafrechtlich sanktioniert. ${ }^{99} \mathrm{Da}-$ rüber hinaus kann der Staat Risiken verlagern, indem er entsprechende Haftungsregeln erlässt um den insoweit geeigneteren oder reicheren Teilnehmer für die Risikoreduktion verantwortlich zu machen. In dieser Perspektive der Risikosteuerung ist die traditionelle Unterscheidung zwischen öffentlichem und privatem Recht praktisch irrelevant. Haftungsregeln, Versicherungsregulierung und sogar Sicherheitsregeln können in beiden Formen erlassen werden. Nur soweit Verwaltungsbehörden

96 Vgl. dazu Homann/Suchanek, Ökonomik (Fn. 92), S. 212 ff.; M. R. Greene, The Government as an Insurer, Journal of Insurance 43 (1976), S. 393-407.

97 Dies wird vertreten von der Assekuranz -Theorie der Steuern, s. F. K. Mann, Steuerpolitische Ideale, Jena 1937 (Nachdr. 1978), S. 106 ff.

98 Dagegen wird es selten möglich sein, im Sinne der Mechanismus-Design-Theorie (vgl. dazu M. J. Holler/G. Illing, Einführung in die Spieltheorie, 6. Aufl., Berlin et al. 2006, S. 340 ff.) durch rechtliche Regelungen Rahmenbedingungen zu schafften, die moral hazard (völlig) ausschließen.

99 Vgl. K. Reus, Das Recht in der Risikogesellschaft, Berlin 2009, S. 71 ff. 
oder der Staat selbst die Durchsetzung übernehmen, bedarf es einer Regulierung durch öffentliches Recht.

Der Staat ist allerdings nicht nur eine notwendige Ergänzung und Gegenkraft zu den Märkten, sondern ebenso eine bedeutende Quelle des Versagens, was deshalb komplementär zum Marktversagen als Staatsversagen bezeichnet wird. ${ }^{100}$ Dabei lässt sich eine Reihe unterschiedlicher Formen risikobezogenen Staatsversagens feststellen. Staatliche Regulierung durch Recht und seine Durchsetzung erzeugen Kosten, die die Kosten des Risikos übersteigen können, ${ }^{101} \mathrm{da}$ fast immer die Reduzierung bestimmter Risiken andere Risiken erhöht oder jedenfalls weniger vermindert. ${ }^{102}$ Wahrnehmungsprobleme können ebenso die Bürokratie und Verwaltung bzw. den Staat insgesamt betreffen. Experten neigen ebenfalls zur Selbstüberschätzung. ${ }^{103}$ Demokratische Verantwortlichkeit politischer Akteure kann die Übertragung der Wahrnehmungsprobleme der allgemeinen Bevölkerung in die staatliche Sphäre bewirken und als Konsequenz entsprechend verfehlte Forderungen nach einer Reduzierung und Regulierung dieser Risiken auslösen. ${ }^{104}$ Im Einzelfall ist es sogar denkbar, dass politische Kräfte und Parteien eine verzerrte Wahrnehmung der Bürger für ihre Zwecke instrumentalisieren, um ihre Ziele durchzusetzen. Sicherheitsgesetze und Atomausstieg bieten Beispiele für eine Mixtur aus problematischen Risikoeinschätzungen und politischer Instrumentalisierung. Außerdem zieht ein positives Handeln des Staates eher Kritik auf sich als Untätigkeit. ${ }^{105}$ Der politische Prozess neigt oft zu einem verfehlten Fokus auf worst case-Szenarien. Die Wahrnehmung der Risiken durch die Bevölkerung und die darauf aufbauende Risikosteuerung hängt sehr stark von dem fragilen Vertrauen in die Experten und in die Staatsgewalt ab. ${ }^{106}$ Risikoreduzierung durch den Staat ist ferner oft ineffizient. ${ }^{107}$ Haftungsregeln können Marktmechanismen verzerren und Versicherungen können den moral hazard fördern. All diese Formen des Staatsversagens machen die Risikosteuerung jedoch nicht überflüssig oder generell schädlich. Im Gegenteil, eine umfassende Risikosteuerung durch den

100 Vgl. H. Hanusch (Hrsg.), Anatomy of Government Deficiencies, Berlin et al. 1983; M. Jänicke, Staatsversagen, München/Zürich 1984, S. 50 ff.; C. Wolf, Markets or Governments. Choosing Between Imperfect Alternatives, 2. Aufl., Cambridg, Mass.: MIT Press 1993, S. 59 ff.

101 Vgl. R. J. Zeckhauser/W. K. Viscusi, Risk within Reason, Science 248 (1990), S. 559-564; auch in: Viscusi, Tradeoffs (Fn. 68), S. 149 ff.; vgl. ferner Viscusi, Risk Policy (Fn. 59), S. 68 ff.

102 Zum generellen Problem der risk tradeoffs vgl. insbesondere A. Wildavsky, No Risk is the Highest Risk of All, American Scientist 67 (1979), S. 32-37; J. D. Graham/J. B. Wiener (Hrsg.), Risk versus Risk, Cambridge, Mass.: Harvard University Press 1995, dort insbes. die beiden Beiträge der Herausgeber S. 1-41 und 226-271.

103 Fischboff, Risk (Fn. 76), S. 35 f.

104 Vgl. R. G. Noll/J. E. Krier, Some Implications of Cognitive Psychology for Risk Regulation, in: C. R. Sunstein (Hrsg.), Behavioral Law and Economics, 2000, S. 325-354 (336 ff.).

105 Viscusi, Risk Policy (Fn. 59), S. 85.

106 Vgl. P. Slovic, Perceived Risk, Trust and Democracy, in: ders., Perception (Fn. 60), S. 316-326.

107 Vgl. R. J. Zeckhauser/W. K. Viscusi, The Risk Management Dilemma, The Annals of the American Academy of Political and Social Science 445 (1996), S. 144-155. 
Staat ist ein unverzichtbares Element jeder Marktwirtschaft, muss aber stets die Möglichkeiten des Staatsversagens mit in Betracht ziehen.

\section{E. Verfassungsrechtliche Direktiven für die staatliche Risikosteuerung}

An dieser Stelle kann lediglich ein allgemeiner und etwas skizzenhafter Überblick des verfassungsrechtlichen Rahmens der Risikosteuerung durch den Staat gegeben werden.

\section{Risikosteuerung als Eingriffsrechtfertigung}

Die Freiheitsrechte des Grundgesetzes begründen eine rechtliche Vermutung dafür, ${ }^{108}$ dass die einzelnen Bürger sowie die privaten Gesellschaften und juristischen Personen die Risiken selbst tragen und die Risikosteuerung eigenverantwortlich übernehmen müssen. Sie können Risiken reduzieren oder verlagern und verteilen, insbesondere durch privatrechtliche Verträge. Die Risikoverlagerung durch Verträge sowie die Risikoverteilung durch alle möglichen Formen privater Versicherungen ist daher allgegenwärtig. Der Staat kann zwar Risiken reduzieren oder neu zuweisen, indem er in die Marktprozesse eingreift, aber jede staatliche Intervention Muss durch legitime und plausible Gründe gerechtfertigt werden und zudem die verfassungsrechtlichen Anforderungen beachten, an deren vorderster Stelle das Verhältnismäßigkeitsprinzip steht. ${ }^{109}$ Risikoreduzierung und die Zwecke der Risiko(re)allokation sind derartige Gründe, die Eingriffe in die Freiheitsrechte prinzipiell rechtfertigen. Das setzt voraus, dass Risiken auch tatsächlich bestehen, wobei die allgemeinen Regeln für Tatsachenfeststellungen und Prognosen anwendbar sind. ${ }^{110}$ In diesem Rahmen wird man auch verlangen können, dass die Erkenntnisse der ökonomischen und psychologischen Wissenschaften über die Wahrnehmungsfehler berücksichtigt werden, soweit sie erkennbar die Risikoeinschätzung beeinflussen. Dabei lassen sich kaum generelle Bedingungen und Regeln aufstellen, welche biases und in welchem Maß diese zu berücksichtigen sind. Allerdings wird man generell plausible und nachvollziehbare Risikoeinschätzungen und -bewertungen verlangen müssen, was eine weitgehende Ausschaltung von biases beinhaltet. Die auf systematischen Wahrnehmungsfehlern begründeten Sorgen der Bürger dürften als Eingriffsrechtfertigung kaum ausreichen.

\section{Verfassungsrechtliche Pflicht zur Risikosteuerung?}

Das Grundgesetz verpflichtet den Staat nicht dazu, Risiken zu verlagern oder zu verteilen. Vielleicht lässt sich eine Ausnahme für den Fall der Sozialversicherung be-

108 Vgl. C. Schmitt, Verfassungslehre (1928), 5. Aufl., Berlin 1970, S. 126, 158, 164.

109 Vgl. hier nur B. Pieroth/B. Schlink, Grundrechte Staatsrecht II, 26. Aufl., Heidelberg 2010, Rn. 203 ff.; A. von Arnauld, Die Freiheitsrechte und ihre Schranken, Baden-Baden 1999, S. 110 ff.

110 Vgl. hier nur K. Schlaich/S. Korioth, Das Bundesverfassungsgericht, 8. Aufl., München 2010, Rn. 532 ff. m.w.N. 
gründen, da diese durch mehrere verfassungsrechtliche Vorschriften legitimiert und zudem durch das allgemeine Sozialstaatsprinzip untermauert wird, das allerdings lediglich eine gewisse Garantie der Institutionen der Sozialversicherung als solcher enthält und allenfalls Einschränkungen des sozialen Versicherungssystems begrenzt, darüber hinaus aber keine detaillierten Direktiven enthält. ${ }^{111}$ Verfassungsrechtliche Schutzpflichten, die überwiegend aus den Grundrechten abgeleitet werden, ${ }^{112}$ sind vorrangig auf eine Reduzierung von Risiken ausgerichtet, was eine nachrangige Risikoverlagerung oder -verteilung als Rechtsfolge nicht ausschließt. Das entspricht der Konzentration auf die Risikoreduzierung seit dem 19. Jahrhundert, wie sie im Polizeirecht zum Ausdruck gelangt. Die Schutzpflicht erfasst danach nicht nur die Abwehr einer aktuellen Rechtsverletzung, sondern ebenso die Abwendung objektiver Risiken, die eine bloß theoretisch vorhersehbare Möglichkeit eines Schadenseintritts beinhalten. Sie begründet damit eine Pflicht zur Risikovorsorge, ${ }^{113}$ die sich sogar prinzipiell auf das im Ergebnis gleichwohl hinzunehmende Restrisiko erstreckt. ${ }^{114}$ Sofern man das Institut der grundrechtlichen Schutzpflicht bejaht, ${ }^{115}$ lassen sich generelle Grenzen der Schutzpflicht praktisch nicht feststellen. Schutzpflichten werden mittlerweile auch geradezu beliebig begründet. Umfang und Grenzen können deshalb lediglich durch gegenläufige verfassungsrechtliche Interessen und Rechtsgüter im Wege eines Ausgleichs begründet werden. ${ }^{116}$

Die Verfassung selbst vernachlässigt die Verlagerung von Risiken und die Verteilung von Risiken im Allgemeinen, während das einfache Recht Regelungen über die Risikoreallokation in erheblichem Umfang enthält. Haftungsregeln wie Versicherungsgesetzgebung bilden große eigenständige Rechtsgebiete.

111 Zu Einzelheiten s. R. Gröschner, in: H. Dreier (Hrsg.), GG-Kommentar, Bd. 2, 2. Aufl., Tübingen 2006, Art. 20 (Sozialstaat), Rn. 44; H. M. Heinig, Der Sozialstaat im Dienst der Freiheit, Tübingen 2008, S. $10 \mathrm{ff} ., 457 \mathrm{ff}$.

112 Vgl. hier nur G. Hermes, Das Grundrecht auf Schutz von Leben und Gesundheit, Heidelberg 1987, S. 43 ff.; J. Isensee, Das Grundrecht als Abwehrrecht und als staatliche Schutzpflicht, in: J. Isensee/P. Kirchhoff (Hrsg.), Handbuch des Staatsrechts, Bd. 5, 1. Aufl,, Heidelberg 1992, 1111 Rn. 86 ff.; J. Dietlein, Die Lehre von den grundrechtlichen Schutzpflichten, 1992, S. 34ff; W. Cremer, Freiheitsgrundrechte, Tübingen 2003, S. 228 ff.; klare Darstellung der staatszweckorientierten und der grundrechtlichen Herleitung bei G. Krings, Grund und Grenzen grundrechtlicher Schutzansprüche, Berlin 2003, S. 85 ff., 102 ff.

113 So Isensee, Abwehrecht (Fn. 112), $\mathbb{1 1 1}$ Rn. 106 ff.; D. Lorenz, Grundrechtsschutz gegen Gefahren und Risiken, in: FS Rupert Scholz, München 2007, S. 325 ff. (330 ff., 333).

114 Hermes, Grundrecht (Fn. 112), S. 236 ff.; Isensee, Abwehrrecht (Fn. 111), \111 Rn. 108, das ist freilich umstritten; a.A. C. Degenhart, Kernenergierecht, 2. Aufl., Köln et al. 1982, S. 147 f.; K. Stern, Staatsrecht III/1, München 1988, S. 953.

115 Prinzipiell ablehnend W. Heun, Funktionell-rechtliche Schranken der Verfassungsgerichtsbarkeit, Baden-Baden 1992, S. $66 \mathrm{ff.}$

116 Schon klar herausgearbeitet von Hermes, Grundrecht (Fn. 112), S. $246 \mathrm{ff}$. 


\section{Geringe Determinationskraft der Verfassung}

Die Verfassung begründet praktisch überhaupt keine expliziten, sondern lediglich mittelbare Verpflichtungen. Selbst die öffentliche Sicherheit als solche und der Schutz vor unmittelbaren Gefahren und Risiken werden durch das Grundgesetz nicht ausdrücklich garantiert, obwohl die Gewährleistung der öffentlichen Sicherheit eine zentrale Funktion des modernen Verfassungsstaats ist. ${ }^{117}$ Diese Funktion wurde stets als selbstverständlich angesehen, allerdings wurde ihre rechtliche Garantie als so schwierig betrachtet, dass davon gänzlich abgesehen wurde. ${ }^{118}$ Das Verfassungsgericht begann erst in den siebziger Jahren, ein allgemeines verfassungsrechtliches Grundrecht auf Sicherheit zu konstruieren, ohne dabei diese Formulierung zu benutzen. ${ }^{119}$ Die staatliche Verpflichtung, das Leben und die physische Integrität zu schützen, wird insoweit aus dem Recht auf Menschenwürde in Verbindung mit dem Recht auf Leben abgeleitet. Gleichwohl ist daraus ein umfassendes Recht auf Sicherheit bisher nicht deduziert worden. ${ }^{120}$ Aus der Eigentumsgarantie des Art. 14 GG ergibt sich kaum mehr. Zwar begründen inzwischen sämtliche Grundrechte Schutzpflichten, ${ }^{121}$ auch Art. 14 GG ist davon nicht ausgenommen. ${ }^{122}$ Der umfassende Anspruch der Schutzpflicht für Leben und Gesundheit bis hin zur Abwendung aller Risiken ist aber kaum ernsthaft auf den Schutz des Eigentums vor jeglichem finanziellen Risiko auszudehnen, da dies unabsehbare Folgen hätte, wenngleich angesichts der Uferlosigkeit des Schutzpflichtkonzepts im Grunde keine prinzipiellen Schranken gegenüber einer solchen Ausweitung bestehen. ${ }^{123}$

Während die Verfassung den Staat fast überhaupt nicht zur Risikosteuerung verpflichtet, ermächtigt sie freilich den Staat zu verschiedenen Formen der Risikosteuerung und räumt weite Entscheidungsspielräume ein, ob und wie der Staat mit Risiken umgehen soll. Das trifft sogar auf die grundrechtlichen Schutzpflichten hinsichtlich

117 Die Studie von Stoll, Sicherheit (Fn. 95) konzentriert sich fast ausschließlich auf die Risikoreduzierung, erwähnt aber immerhin die staatliche Unfallversicherung, S. $31 \mathrm{ff}$.

118 Einige ursprüngliche Verfassungen der Staaten der USA erwähnen die öffentliche Sicherheit, so z.B. die Virginia Bill of Rights of $1776, \mathbb{S} 3$.

119 Diesen Begriff prägte Isensee, Grundrecht (Fn. 2), S. 27 ff.; s.a. G. Robbers, Sicherheit als Menschenrecht, Baden-Baden 1987, S. $121 \mathrm{ff}$.

120 Die Außerachtlassung einer Garantie der Sicherheit hat Wissenschaftler nicht davon abgehalten, eine solche konstitutionelle Garantie, die nicht auf das an sich schon problematische Recht auf Sicherheit beschränkt ist, zu konstruieren, s. insbes. M. Möstl, Die staatliche Garantie für die öffentliche Sicherheit und Ordnung, Tübingen 2002, S. 24-28, 37-146; kritisch Krings, Grund (Fn. 112), S. 172 (bloße Addition einzelner Schutzpflichten).

121 BVerfGE 92, 26 (46).

122 BVerfG (3. Kammer des Ersten Senats), NJW 1998, S. 3264 (3265); s. auch P. Szcekella, Die sogenannten grundrechtlichen Schutzpflichten im deutschen und europäischen Recht, Berlin 2002, S. 336., allerdings mit dem verfehlten Verweis auf BVerfGE 97, 350 (377); vgl. ferner Krings, Grund (Fn. 112), S. $177 \mathrm{ff}$.

123 Zwar schützt Art. 14 GG nur das Eigentum und nicht das Vermögen, da letztlich finanzielle Risiken indes auch auf das Eigentum durchschlagen, begrenzt diese Differenzierung im Ergebnis die Schutzpflicht bezüglich Art. 14 GG konstruktiv ebenfalls nicht. 
der Gefährdung der Freiheitsrechte durch private dritte Parteien zu, da sie in der Regel den Staat nicht dazu verpflichten, die Bürger mit ganz bestimmten Maßnahmen zu schützen. Im Allgemeinen verpflichten die Schutzpflichten den Staat nur dazu, überhaupt zu handeln, aber nicht dazu, in einer bestimmten Weise zu handeln, wenngleich die Rechtsprechung zunehmend zur Einengung der Spielräume tendiert. Zugleich rechtfertigen die Grundrechte Dritter sowie die allgemeinen verfassungsrechtlichen Prinzipien weitreichende Eingriffe in die Freiheitsrechte des Einzelnen durch staatliche Regulierungen. Wenn man die verfassungsrechtlichen Vorgaben des Grundgesetzes hinsichtlich der Risikosteuerung in einem Satz zusammenfassen will, kann man sagen, dass das Grundgesetz weite Ermächtigungen ohne Verpflichtungen enthält. Es ermächtigt den Staat dazu, ein soziales Versicherungssystem einzurichten und zu regulieren und das Sozialstaatsprinzip rechtfertigt erhebliche Eingriffe in die Individualrechte des Einzelnen. Dieses Prinzip schützt das etablierte Sozialversicherungssystem gegen eine vollständige Abschaffung, aber nicht gegen fundamentale Modifizierungen, obwohl die einzelnen bereits erworbenen individuellen Ansprüche bis zu einem gewissen Grad auch durch das Eigentumsgrundrecht des Art. 14 GG gegen Entzug geschützt sind. ${ }^{124}$

Meistens ist der Schutz gegen Eingriffe von dritter Seite jedoch noch schwächer ausgeprägt. Die allgemeine Ermächtigung zum Erlass von Zivilrecht erlaubt die Risikoverlagerung durch Haftungsregelungen, indem entweder die Haftung der Privatrechtssubjekte begründet, begrenzt oder verlagert wird. Die Ermächtigung verpflichtet den Staat aber ebenso wenig wie sie den Einzelnen schützt. Die Verfassung begrenzt die Reallokation von Risiken durch Steuern oder Versicherungen in keiner substantiellen Weise und verpflichtet den Staat auch in dieser Hinsicht nicht. Im übrigen Muss die Rechtsordnung selbst Rechtssicherheit gewährleisten, aber sie stiftet nicht Vorhersehbarkeit im Hinblick auf die Risiken dieser Welt. Das würde das Recht und die Verfassung im Besonderen maßlos überanstrengen.

Der Überblick über den verfassungsrechtlichen Rahmen offenbart eine große Diskrepanz zwischen der aktuellen Rolle und Bedeutung des Staates bei der Risikosteuerung auf der einen Seite, die kaum zu überschätzen ist, und der Widerspiegelung dieser Rolle in der Verfassung auf der anderen Seite. Die Knappheit und Armut der Regelungen, die geringe Determinierung der Risikosteuerung durch verfassungsrechtliche Direktiven ist allerdings gleichwohl nicht erstaunlich, wenn man die große Bandbreite der Probleme der Risikosteuerung ins Auge fasst. Es gibt fast unbegrenzte Entscheidungsspielräume für den Staat, Risiken zu reduzieren, Risiken zu regulieren sowie durch Haftungsregeln oder Besteuerungen zu verlagern, Risiken durch die Schaffung staatlicher Versicherungssysteme oder durch die Begründung von Ver-

124 Vgl. J. Wieland, in: H. Dreier (Hrsg.), GG-Kommentar, Bd. 1, 2. Aufl., Tübingen 2004, Art. 14 Rn. $61 \mathrm{ff}$. 
pflichtungen zu verteilen, sich an privat organisierten Versicherungen zu beteiligen. Die verfassungsrechtliche Determinierung des gesamten Bereichs der Risikosteuerung ist vergleichsweise schwach. ${ }^{125}$ Diese Situation wird gerechtfertigt durch die außerordentliche Komplexität der Risikosteuerung wie auch durch die Tatsache, dass das Verfassungsgericht über keine spezifischen Fähigkeiten verfügt, Risiken einzuschätzen oder Direktiven für die Risikosteuerung zu geben. Die schwache Determinierung dieser Risikosteuerung durch die Verfassung ist insoweit notwendig und nicht zu beklagen. Es wäre verfehlt, aus den Normen der Verfassung, insbesondere auch aus den grundrechtlichen Schutzpflichten, weiterreichende Vorgaben für die Risikosteuerung ableiten zu wollen, auch wenn dies weiten Teilen der Staatsrechtslehre widerstreben mag.

125 Rechtspolitisch wird dieser Rahmen ausgefüllt durch die Diskussion um einen (liberalen) Paternalismus: R. H Thaler/C. R. Sunstein, Libertarian Paternalism Is Not an Oxymoron, Univ. of Chicago Law Review 70 (2003), S. 1159-1202; dies., Nudge. Improving Decisions about Health, Wealth, and Happiness, 2008; H. Eidenmüller, Liberaler Paternalismus, JZ 2011, S. 814-821. 